\title{
Special Issue on New Paradigms of Software Production and Deployment
}

\author{
Alfredo Capozucca $\cdot$ Jean-Michel Bruel ${ }^{2} \cdot$ Manuel Mazzara $^{3} \cdot$ Bertrand Meyer $^{4,5}$
}

Published online: 13 January 2022

(c) The Author(s), under exclusive licence to Springer Nature Singapore Pte Ltd 2022

\section{The DEVOPS Series in the Château de Villebrumier: 2018-19}

It was March 5th, 2018 when the first workshop of the DEVOPS series was held at the new LASER center at the Château de Villebrumier, near Montauban and Toulouse, France.

The LASER center is inspired by the prestigious precedent of the Dagstuhl center in Germany (the model for all such ventures), but adding its own sunny touch of accent du sud-ouest (the tuneful tones of Southwest France). The chateaux is also the Grand Quartier Général of the LASER foundation , ${ }^{1}$ the non-profit organisation behind the establishment of the popular LASER summer school in Elba, Italy. It is meant to be a venue for high-tech events of a few days to a week in a beautiful setup in the midst of a region rich with historical, cultural, and culinary attractions.

The DEVOPS series organised at the LASER center was (and it is still meant to be) a venue for in-depth discussions about new development and deployment methods coined under the term "DevOps", and their impact on traditional software engineering methods.

\footnotetext{
Alfredo Capozucca

alfredo.capozucca@uni.lu

Jean-Michel Bruel

jean-michel.bruel@irit.fr

Manuel Mazzara

m.mazzara@innopolis.ru

Bertrand Meyer

Bertrand.Meyer@inf.ethz.ch

University of Luxembourg, Esch-sur-Alzette, Luxembourg

2 University of Toulouse, Toulouse, France

3 Innopolis University, Innopolis, Russia

4 Schaffhausen Institute of Technology, Neuhausen am Rheinfall, Switzerland

5 Innopolis University, Innopolis, Russia
}

As said, this series started in 2018 with the organisation of the first workshop. Although this workshop was not the absolute first event organised in the LASER center, it was the first time that submissions were opened to everyone without any invitation. It was a good occasion not only to strengthen already existing collaborations, but also to meet new people and develop new collaborations. A proof of that, it is this topical issue. The event was repeated in May 6-8, 2019, at the same venue.

For each workshop, a collection of revised selected papers were published in a sub series of the Springer Lecture Notes in Computer Science series ([2, 3]).

\section{The Topical Issue: $2020-21$}

There was an initial plan to run a third event of the series in Spring 2020, but it had to be cancelled due to the COVID-19 pandemic. After initial consideration to run it as an online event in late 2020/early 2021, it was decided to postpone it until the situation would allow us to come back to the original settings.

Instead, a journal Topical Issue was organised to complete the trilogy and serve as a replacement to guarantee continuity. Thus, the aim and scope of this topical issue remained aligned with those of the DEVOPS series: to provide the most recent stages of what is being done in the area of software engineering targeting modern software development methods, with particular interest at those aimed at speeding up the development life cycle, while ensuring that the product to be released meets the expected levels of quality.

Of course, the organisation and review process did also suffer from the effects caused by the pandemic. The initial timeline had to be adapted to grant extensions to authors to submit their work, then to reviewers to come up with their reviews, and us (the guest editors) to make the final decisions. Despite of that, the full process was completed as

\footnotetext{
$\overline{1}$ http://laser-foundation.org.
} 
initially promised in 2021 , leading to accepted papers to be published between August and November.

We received 13 submissions, from which finally 6 made it into the Topical Issue. Each submission was reviewed for two independent reviewers following a two-round revision process: i.e. authors of papers that required major modifications had the chance to incorporate reviewers' feedback into the second submission. Each submission had to have at least one reviewer accepting the paper to be considered by us (guest editors) to make the final decision: either accepting or rejecting the submission.

The Topical Issue on "New paradigms of software production and deployment", is now published by Springer Nature (SN) Computer Science. SN Computer Science is a broad-based, peer reviewed journal that publishes original research in all the disciplines of computer science, including various inter-disciplinary aspects.

\section{Articles in the Topical Issue}

This Topical Issue [4] contains the following papers, accepted after careful peer-reviewing:

- Applying Model-Driven Engineering to Stimulate the Adoption of DevOps Processes in Small and MediumSized Development Organizations, by [9]: in this paper, authors report the challenges that small and mediumsize companies face with implementing microservices. Authors propose a model-driven workflow to cope with these challenges, which is illustrated and validated via an open-source case study.

- Monitoring a CI/CD Workflow Using Process Mining, by [8]: this papers describes how data generated by the execution of $\mathrm{CI} / \mathrm{CD}$ pipelines can be used to monitor (and then gain better understanding) about how the current pipelines are working. The approach proposed by the authors relies on the implementation of process mining algorithms into a well known open source distributed streaming platform. This approach is illustrated through its implementation in an European company operating in the global market.

- Closing the Feedback Loop in DevOps Through Autonomous Monitors in Operations, by [7]: the contributions brought by this paper belong to the area of artificial intelligence for IT operations. More particularly, authors describe how to use machine learning techniques to enhance the quality of the feedback going from operations to development. These contributions are presented and validated through an industrial case study.

- Virtualization Costs: Benchmarking Containers and Virtual Machines Against Bare-Metal, by [5]: in this paper, authors focus on one of the most relevant assets used by
DevOps practitioners: virtualisation. After having retrieved the most popular virtualisation technologies available in the market, authors perform a resource-oriented benchmark study to determine the overhead introduced by each virtualisation technology.

- SecDocker: Hardening the Continuous Integration Workflow, by [6]: this study addresses security issues in DevOps environments that rely on containers. Authors present a novel open-source tool aimed at handling the reported security threats and vulnerabilities.

- Software Product System Model: A Customer-Value Oriented, Adaptable, DevOps-Based Product Model, by [1]: authors of this paper present a customer-value-oriented software product management model that covers the complete software development life cycle. Authors also report the insights found after a 2 years evaluation period of such a model in the context of a large industrial case study.

This Topical Issue promotes studies that fall into the context of DevOps and which are based on clear and solid evidence. Thus, we trust the advantages (and/or limitations) of the insights brought by these studies make possible to advance the knowledge on the targeted field.

We hope you will enjoy reading and find it useful for your future work.

Acknowledgements We want to thank not only the authors who submitted articles to the topical issue, but also those who acted as reviewer. Beside submitted article's authors, other colleagues also helped in the review process. Our thanks to all of them for their disinterested participation to help us reach the expected high quality contribution level. We finally want to thank the Editor-in-Chief and the rest of the editorial team.

Author Contributions All authors have equally contributed in the production both of this editorial and topical issue.

Funding Information Not applicable.

Availability of Data and Materials Not applicable.

Code Availability Not applicable.

\section{Declarations}

Conflict of Interest The authors declare that they have no conflict of interest.

Code Availability Not applicable.

\section{References}

1. Altunel H, Say B. Software product system model: a customervalue oriented, adaptable, devops-based product model. SN Comput Sci. 2022. https://doi.org/10.1007/s42979-021-00899-9. 
2. Bruel J, Mazzara M, Meyer B. Software engineering aspects of continuous development and new paradigms of software production and deployment-first international workshop, DEVOPS 2018, Chateau de Villebrumier, France, March 5-6, 2018, Revised Selected Papers, Lecture Notes in Computer Science, vol 11350. Springer. 2019. https://doi.org/10.1007/978-3-030-06019-0.

3. Bruel J, Mazzara M, Meyer B. Software engineering aspects of continuous development and new paradigms of software production and deployment - second international workshop, DEVOPS 2019, Château de Villebrumier, France, May 6-8, 2019, Revised Selected Papers, Lecture Notes in Computer Science, vol 12055. Springer. 2020. https://doi.org/10.1007/978-3-030-39306-9.

4. Capozucca A, Bruel J, Mazzara M, et al (eds), New paradigms of software production and deployment, Springer, 2021. https:// link.springer.com/journal/42979/topicalCollection/AC_84b9b 1015f30a3731b3e5c9e410a11a7

5. Giallorenzo S, Mauro J, Poulsen M, et al. Virtualization costs: benchmarking containers and virtual machines against bare-metal. SN Comput Sci. 2021. https://doi.org/10.1007/ s42979-021-00781-8.
6. González D, Rodríguez Lera F, Esteban G, et al. Secdocker: hardening the continuous integration workflow. SN Comput Sci. 2022. https://doi.org/10.1007/s42979-021-00939-4q.

7. Hrusto A, Runeson P, Engström E. Closing the feedback loop in devops through autonomous monitors in operations. SN Comput Sci. 2021. https://doi.org/10.1007/s42979-021-00826-y.

8. Nogueira AF, Zenha-Rela M. Monitoring a ci/cd workflow using process mining. SN Comput Sci. 2021. https://doi.org/10.1007/ s42979-021-00830-2.

9. Sorgalla J, Wizenty P, Rademacher F, et al. Applying modeldriven engineering to stimulate the adoption of devops processes in small and medium-sized development organizations: the case for microservice architecture. SN Comput Sci. 2021. https://doi. org/10.1007/s42979-021-00825-z.

Publisher's Note Springer Nature remains neutral with regard to jurisdictional claims in published maps and institutional affiliations. 